\title{
Critical analysis of the major and ancillary imaging features of LI-RADS on 127 proven HCCs evaluated with functional and morphological MRI: Lights and shadows
}

\author{
Vincenza Granata ${ }^{1}$, Roberta Fusco ${ }^{1}$, Antonio Avallone ${ }^{2}$, Francesco Filice ${ }^{1}$, Fabiana \\ Tatangelo ${ }^{3}$, Mauro Piccirillo ${ }^{4}$, Roberto Grassi ${ }^{5}$, Francesco Izzo $^{4}$ and Antonella Petrillo ${ }^{1}$ \\ ${ }^{1}$ Department of Radiology, "Istituto Nazionale Tumori, IRCCS, Fondazione G. Pascale", Naples, Italy \\ ${ }^{2}$ Department of Abdominal Oncology, "Istituto Nazionale Tumori, IRCCS, Fondazione G. Pascale", Naples, Italy \\ ${ }^{3}$ Department of Diagnostic Pathology, "Istituto Nazionale Tumori, IRCCS, Fondazione G. Pascale", Naples, Italy \\ ${ }^{4}$ Department of Abdominal Surgical Oncology, Hepatobiliary Unit, "Istituto Nazionale Tumori, IRCCS, Fondazione G. Pascale", \\ Naples, Italy \\ ${ }^{5}$ Department of Radiology, Second University of Naples, Piazza Miraglia, Naples, Italy \\ Correspondence to: Roberta Fusco, email: r.fusco@istitutotumori.na.it \\ Keywords: HCC, Li-RADS, magnetic resonance imaging, diffusion weighted imaging, dynamic contrast assessment \\ Received: February 13, $2017 \quad$ Accepted: March 22, $2017 \quad$ Published: April 19, 2017 \\ Copyright: Granata et al. This is an open-access article distributed under the terms of the Creative Commons Attribution License \\ 3.0 (CC BY 3.0), which permits unrestricted use, distribution, and reproduction in any medium, provided the original author and \\ source are credited.
}

\section{ABSTRACT}

Purpose: To report a critical analysis of major and ancillary MR imaging features in assessment of HCC.

Methods: Retrospectively we evaluated 70 cirrhotic patients with 173 nodules, which were subjected to MR study at 0 time (MR0), after 3 (MR3) and 6 months (MR6) using two different contrast media. EOB-GD-DTPA was injected at MRO and MR6, while Gd-BTD03A at MR3. Three expert hepatic radiologists reviewed all images, recording, according to LI-RADS, the size, the presence and quality of arterial-phase hyperenhancement, washout and capsule appearance, threshold growth. Additionally, we recorded signal intensity (SI) on T2-W images, on DWI, on apparent diffusion coefficient (ADC) maps and SI on T1-W images of EOB-GD-BPTA hepatospecific phase. Median value of ADC and of Intravoxel incoherent motion related parameters were assessed.

Results: 127 HCCs and 24 dysplastic nodules were assessed. Hypervascular on arterial phase was found in 84 HCCs, washout appearance in 124, capsule appearance in 111, hypointensity on hepatospecific phase in 127, hyperintensity on T2-W sequences and restricted diffusion in 107. Hyper vascular on arterial phase was found in 17 dysplastic nodules, wash-out appearance in 2, hypointensity on hepatospecific phase in 7 while no dysplastic nodules showed capsule appearance, hyperintensity on T2-W and restricted diffusion. Highest accuracy was obtained by washout appearance and hypointense signal on hepatospecific phase (97\% and $95 \%$ ).

Conclusions: Hypointensity on hepatospecific phase and washout appearance are the most relevant diagnostic sign for differentiating low-risk from high-risk HCC nodules. The capsule appearance, $\mathrm{T2}-\mathrm{W}$ hyperintensity and restricted diffusion have high positive predictive value.

\section{INTRODUCTION}

Liver Imaging Reporting and Data System (LIRADS) is a score to report and interpret hepatic imaging characteristics on computed tomography (CT) and magnetic resonance (MR) studies in patients with risk for hepatocellular carcinoma (HCC) [1-2]. European Association for the Study of the Liver (EASL), in accordance with the guidelines of the American Association for the Study Liver Diseases (AASLD), recommended that in order 
to characterize HCC, non-invasive criteria can exclusively be applied to cirrhotic patients using 4-phase multidetector CT scan or dynamic contrast-enhanced MR examination [3]. Diagnosis is based on the identification of the HCC typical mark (hypervascular in the arterial phase and washout in the portal venous or delayed phases) [3]. Considering that the imaging characteristics used to identify hepatic lesions, could lead to uncertainty in characterization and absence of reproducibility both in clinical care and in research [4], American College of Radiology (ACR) sustained the development of LI-RADS in order to standardize the interpreting, reporting and data collection of HCC imaging. LI-RADS system meets the necessity to perform an exact definition of $\mathrm{HCC}$, in fact it is clinically significant to discriminate between HCC and other malignancies such as cholangiocarcinoma or benign nodules, because the management changes substantially [1]. Moreover, current systems neglect the criteria for vascular invasion diagnosis by HCC, which has important implications in staging and treatment [2]. In the current (v. 2014) LI-RADS [5], the HCC diagnosis was done primarily on the presence/absence of major imaging features. Major imaging features are used to categorize LR-3, LR-4, and LR-5; these comprise arterial-phase hyperenhancement, tumor diameter, washout appearance, capsule appearance and threshold growth [5]. Ancillary imaging features could be employed to change the LI-RADS category $[1,5]$. Ancillary features giving preferentiality to malignancy (diffusion restriction, moderate T2 hyperintensity, T1 hypointensity on hapatospecifc phase) could be used to upgrade system by one or more categories, but not beyond LR-4 [1, 5-8]. In contrast, ancillary features favoring benignity can be used to decrease category down to LR-1 [5]. Our purpose is to report a critical analysis of MR Imaging major and ancillary features to assess HCC smaller than $20 \mathrm{~mm}$.

\section{RESULTS}

Among 173 nodules, eight were combined hepatocellular-cholangiocarcinoma, seven metastases, 7 cirrhotic regenerative nodules, 24 dysplastic nodules and 127 HCCs (median 1.8 lesions for patients). All HCCs were histologically classified according to the major Edmondson-Steiner grade on final pathologic reports as follows: grade $1(n=30)$, grade $2(n=61)$, grade $3(n=$ $36)$, and grade $4(\mathrm{n}=0)$.

We does not evaluated the imaging features of combined nodules, metastases and cirrhotic regenerative nodules while we analyzed HCCs and dysplastic nodules.

We analyzed only lesions with a diameter between 12 and $20 \mathrm{~mm}$.

When we evaluated T2-W images, arterial phase, portal phase and hepatospecific phase of contrast study we found difference of measured lesion size on different sequences, but this is not statistically significant (differences median value is $0.2 \mathrm{~mm}$, range $0.1-0.3 \mathrm{~mm}$; $\mathrm{p}$ value $>0.05$ at Kruskal Wallis test). So we reported a median value of lesion size measured by 4 sequences that was $18 \mathrm{~mm}$ (range 12- $20 \mathrm{~mm}$ ) (Figure 1).

84 HCCs (13 grade 1, 42 grade 2 and 29 grade 3 ) had typical hallmark (hypervascular in the arterial phase with washout in the portal and equlibrium phases) (Figure 2); 40 (15 grade 1, 18 grade 2 and 7 grade 3 ) nodules were no hypervascular in arterial phase, but had washout appearance (atypical nodules) (Figure 3). We found the capsule appearance in $111(89,5 \%)$ nodules.

Additionally, we detected 15 nodules only during hepatospecific phase. Among them 3 (20\%) were HCCs (2 grade 1 and 1 grade 2), 5 (33,3\%) dysplastic nodules and $7(46,7 \%)$ cirrhotic regenerative nodules.

All 127 HCCs were hypointense on hepatospecific phase (Figure 4).

Among the $3 \mathrm{HCC}$ detected by hepatospecific phase, one (grade 2) at MR6 became hypervascular on arterial phase (Figure 5) with wash-out on portal phase. The signal was isointense on T2-W and DWI.

No nodule grew during follow up.

All typical nodules were hyperintense on T2-W and DWI, with diffusion restriction and hypointense signal on ADC maps, 23 (57,5\%) out of atypical nodules were hyperintense on T2-W and DWI, with diffusion restriction and hypointense signal on ADC maps, while 17 (42,5\%) were iso-hypointense on T2-W and DWI and isointense on ADC maps. The median value for ADC was $1,47 \times 10^{-3}$ $\mathrm{mm}^{2} / \mathrm{s}$ (range $0,94-2,44 \times 10^{-3} \mathrm{~mm}^{2} / \mathrm{s}$ ), for fp was $33,3 \%$ (range 12,14-54,08\%), for Dp 45,3 x10 $0^{-3} \mathrm{~mm}^{2} / \mathrm{s}$ (range 13,7$52,7 \times 10^{-3} \mathrm{~mm}^{2} / \mathrm{s}$ ) and for Dt $0.9 \times 10^{-3} \mathrm{~mm}^{2} / \mathrm{s}$ (range $0.81-$ $1,51 \times 10^{-3} \mathrm{~mm}^{2} / \mathrm{s}$ ). Among typical and atypical nodules we found an overlapping of ADC and IVIM derived parameters values. We not found difference significant statistically between typical and atypical nodules using ADC or IVIM parameters values ( $\mathrm{p}$ value $<0.05$ at Kruskal Wallis test).

Among 24 dysplastic nodules, 5 (20,8 \%) were detected only during hepatospecific phase. Seventeen lesions $(70,8 \%)$ were isointense on $\mathrm{T} 2-\mathrm{W}, \mathrm{DW}$ and ADC map with hyperenhancement during arterial phase and isointense signal during portal, equilibrium and hepatospecific phase of contrast studies. Two lesions $(8,3 \%)$ were isointense on T2-W, DW, ADC map and hypointense on arterial, portal and hepatospecific phase of contrast studies (Figure 6).

We evaluated 210 arterial phases (70 at MR0, 70 at MR3 and 70 at MR6) to assess the image quality degradation. The median score for all arterial phase with Gd-BT-DO3A (70 studies) was 1, while the median score for all arterial phase with GD-EOB-DTPA (140 studies) was 3 (range 1-4); there was significant statistically difference between the quality on arterial phase with GdBT-DO3A and the quality on arterial phase with GD-EOBDTPA ( $p$ value $=0.03$ at Kruskal Wallis test). In $25(17,8$ $\%$ ) cases the images, during arterial phase with GD-EOBDTPA, were uninterpretable (Figure 7). 


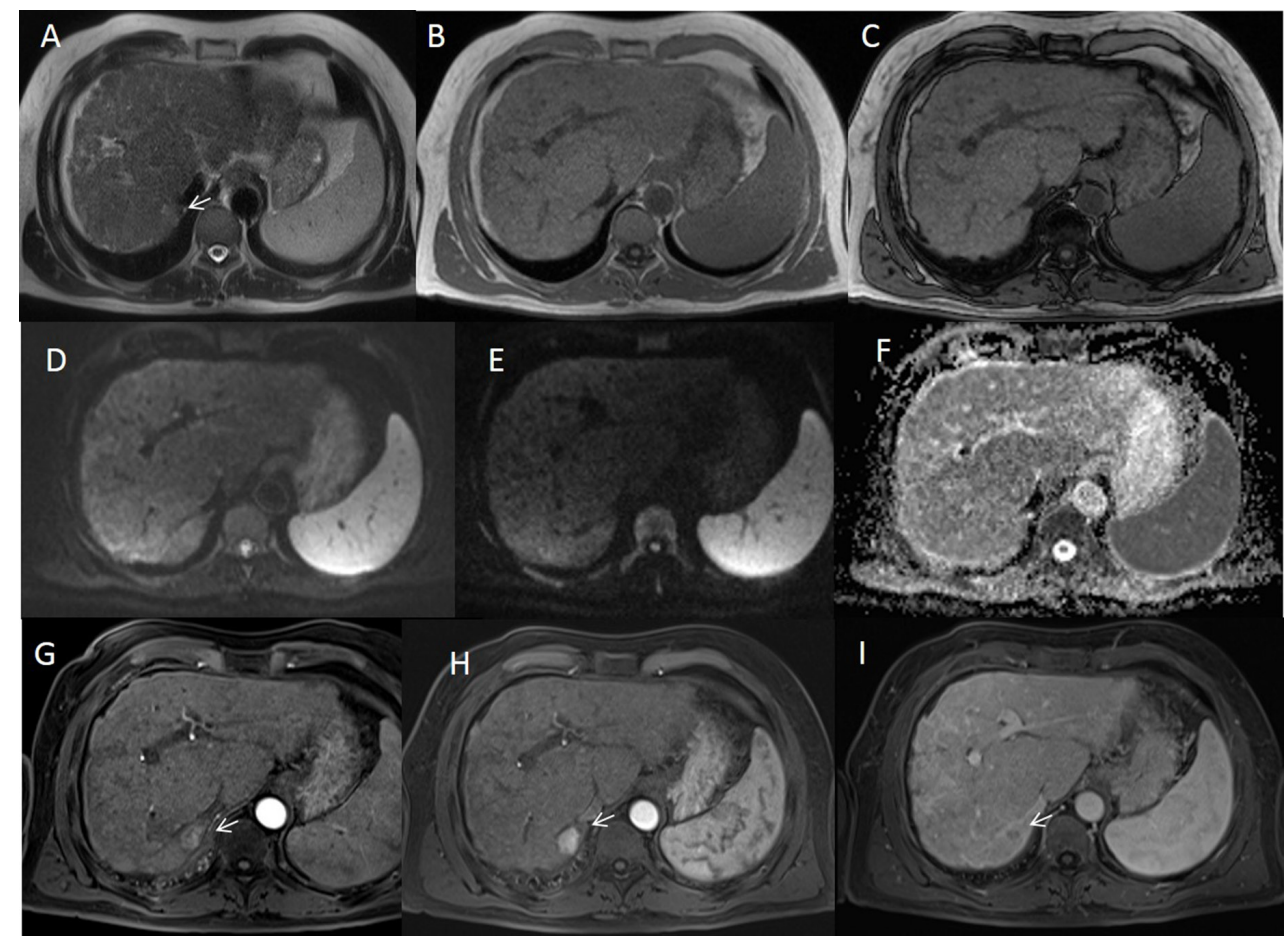

Figure 1: Man sixty-five years old with HCC on VII hepatic segment. The lesion appears hyperintense (arrow) on HASTE T2-W axial plane image sequence $\mathbf{A}$ while on in-of-phase T1-W and out of phase T1-W sequences (B and $\mathbf{C}$ ) the lesion is isointense. On DW images ( $\mathbf{D}$ b0 s/mm² DW image, $\mathbf{E}$ b800 s/mm² DW image, F ADC map) the HCC shows isointense signal. During arterial phase (G, $\mathbf{H}$ and I) the HCC is hypervascular (arrow) with wash-out in portal phase (arrow).

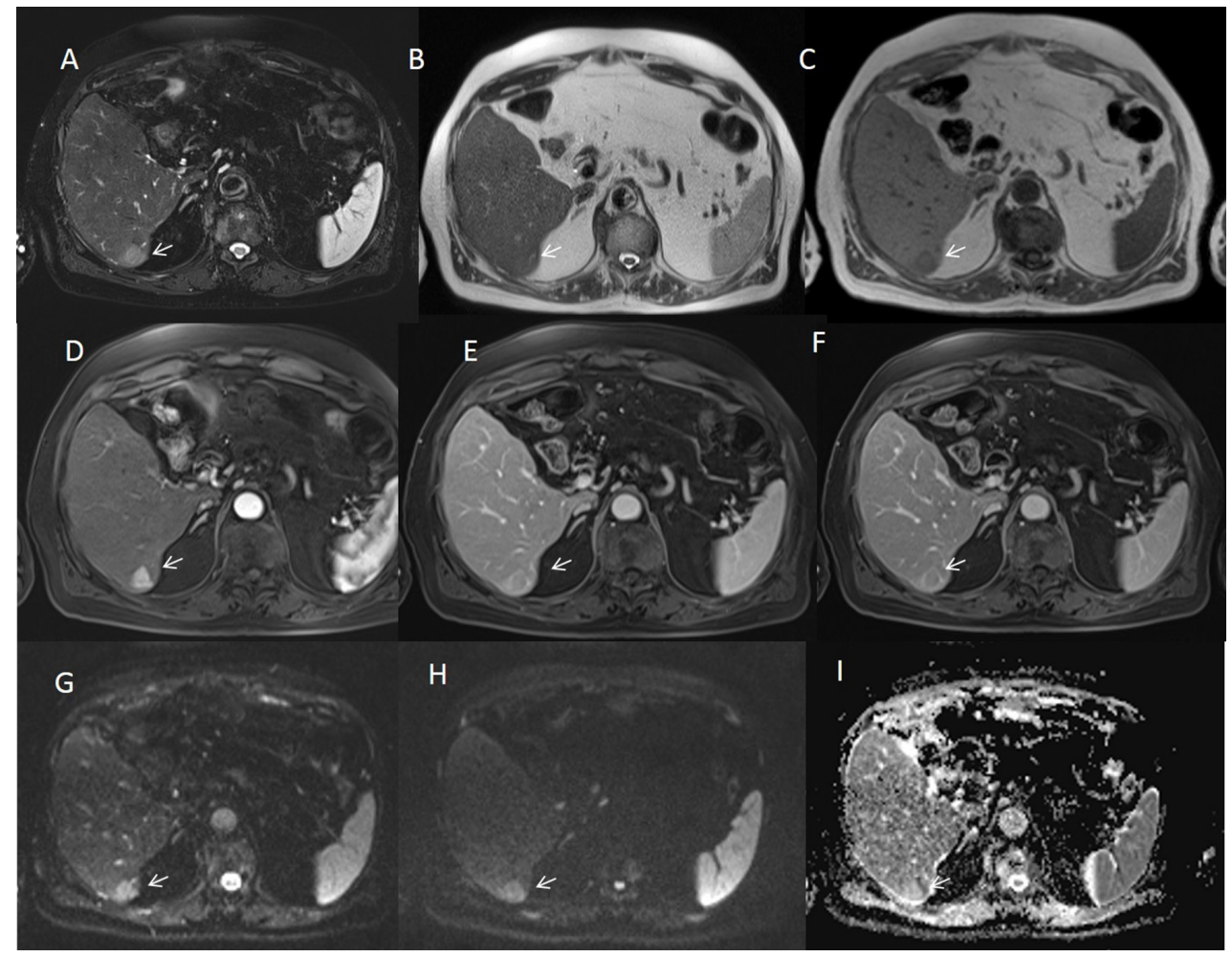

Figure 2: Man seventy-three years old with typical HCC on VI hepatic segment. The HCC is hyperintense (arrow) on T2-W sequences (A and B), hypointense (arrow) on T1-W (C) sequences, hyper vascular (arrow) on arterial phase (D), with wash-out appearance (arrow) on portal phase (E) and capsule appearance (arrow) on equilibrium phase (F) of contrast study with Gd-BT-DO3A. The HCC shows (arrows) restrict diffusion (G b50 s/ $\mathrm{mm}^{2}$ DW image, $\mathbf{H}$ b800 s/mm² DW image, I ADC map). 


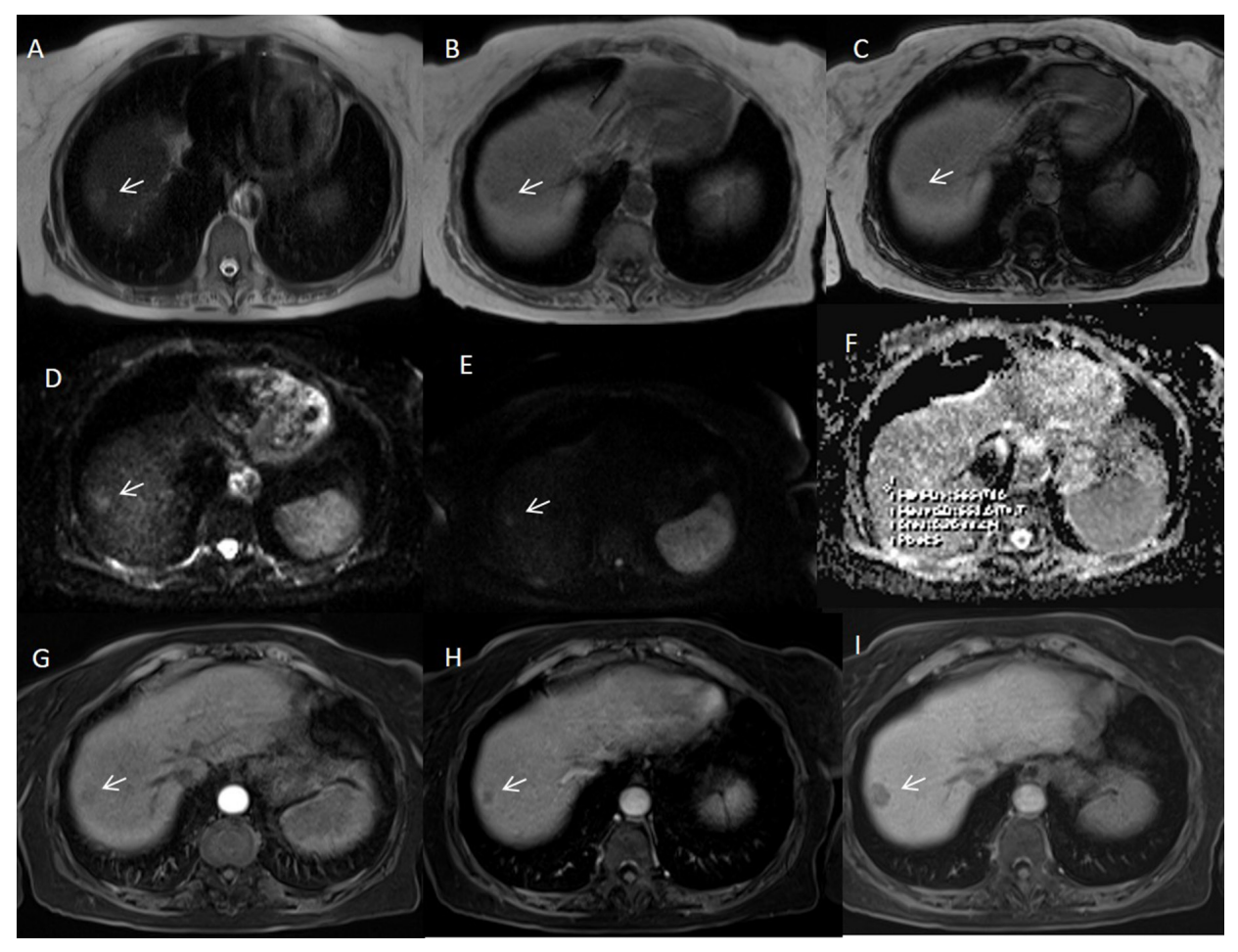

Figure 3: Woman fifty-three years old with atypical HCC on VII-VIII hepatic segment. The HCC is hyperintense (arrow) on T2-W sequences (A) and hypointense (arrow) on T1-W in-of- phase and out-of-phase sequences (B and $\mathbf{C}$ out-of-phase). The lesion shows (arrows) restrict diffusion (D b50 s/ $\mathrm{mm}^{2}$ DW image, $\mathbf{E}$ b800 s/ $\mathrm{mm}^{2}$ DW image, F ADC map). During arterial phase (G), it is not hypervascular (arrow) appearance, while there is wash-out appearance (arrow) on portal phase (H) and capsule appearance (arrow) on equilibrium phase (I) of contrast study with Gd-BT-DO3A.

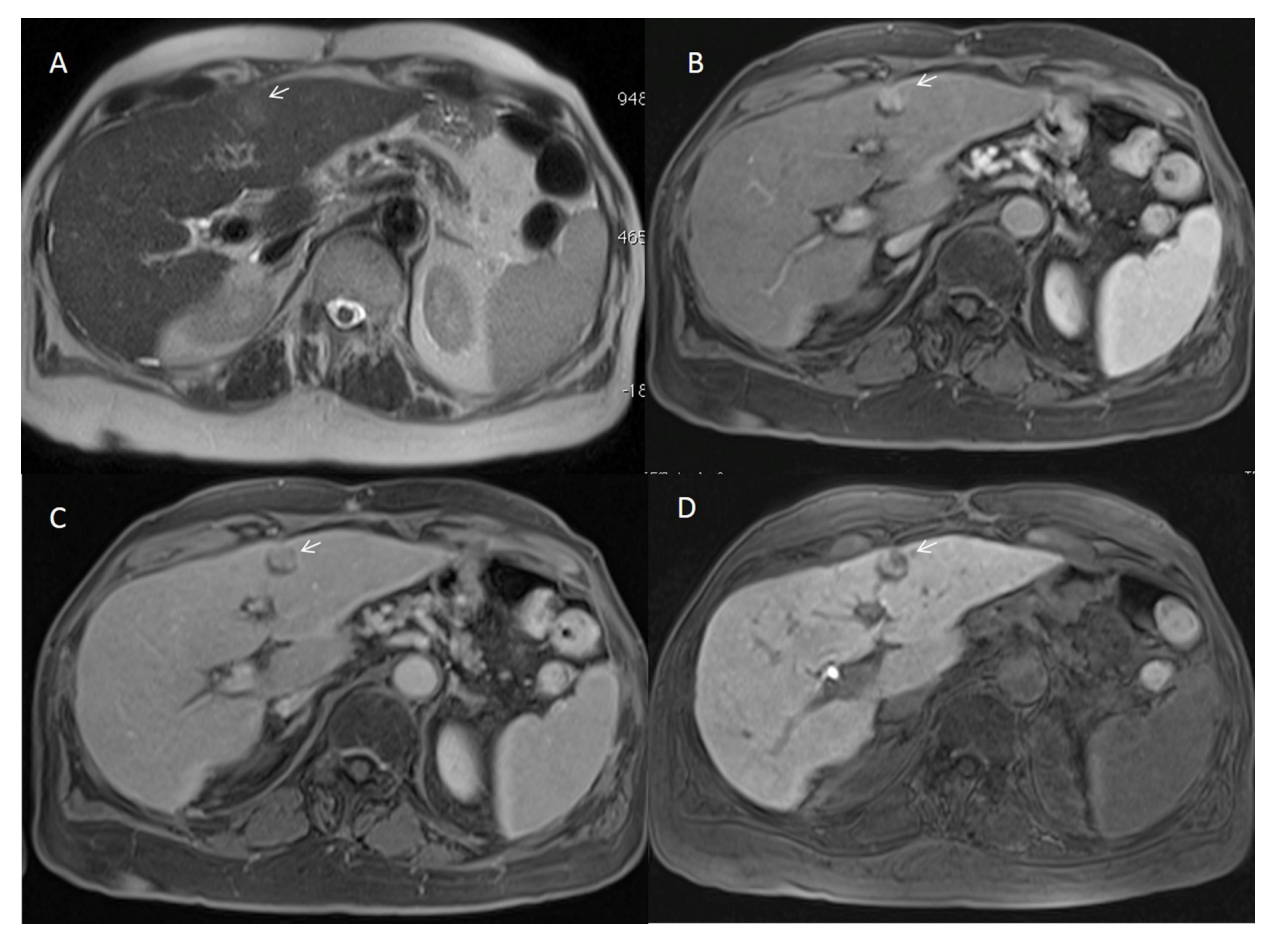

Figure 4: Man sixty-one years old with nodule of HCC in dysplastic nodule on II hepatic segment. The nodule is hyperintense (arrow) on T2-W sequences (A), hyper vascular (arrow) on arterial phase (B), isointense on portal phase (C) with a peripheral hypointense signal (arrow) on hepatospecific phase (D) of contrast study with EOB-GD-DTPA. 


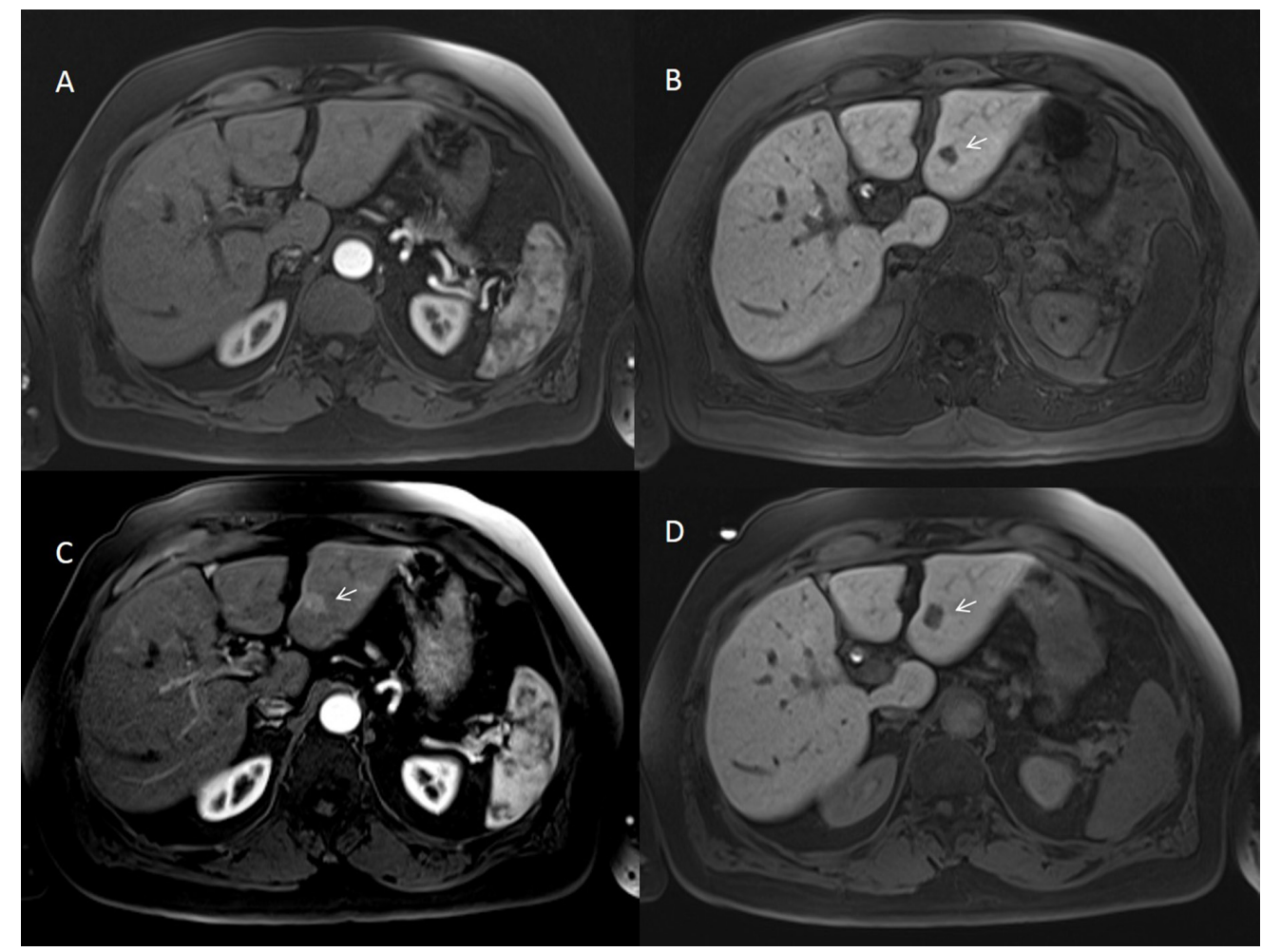

Figure 5: Man seventy-four years old with HCC on III hepatic segment. At MR0 study with EOB-GD-DTPA the lesion is not hypervascular on arterial phase (A) and it is evident on hepatospecific phase (B) of contrast study (arrow). At MR6 study with EOB-GDDTPA, the HCC is hypervascular (arrow) on arterial phase (C) with hypointense signal (arrow) on hepatospecific phase (D) of contrast study.

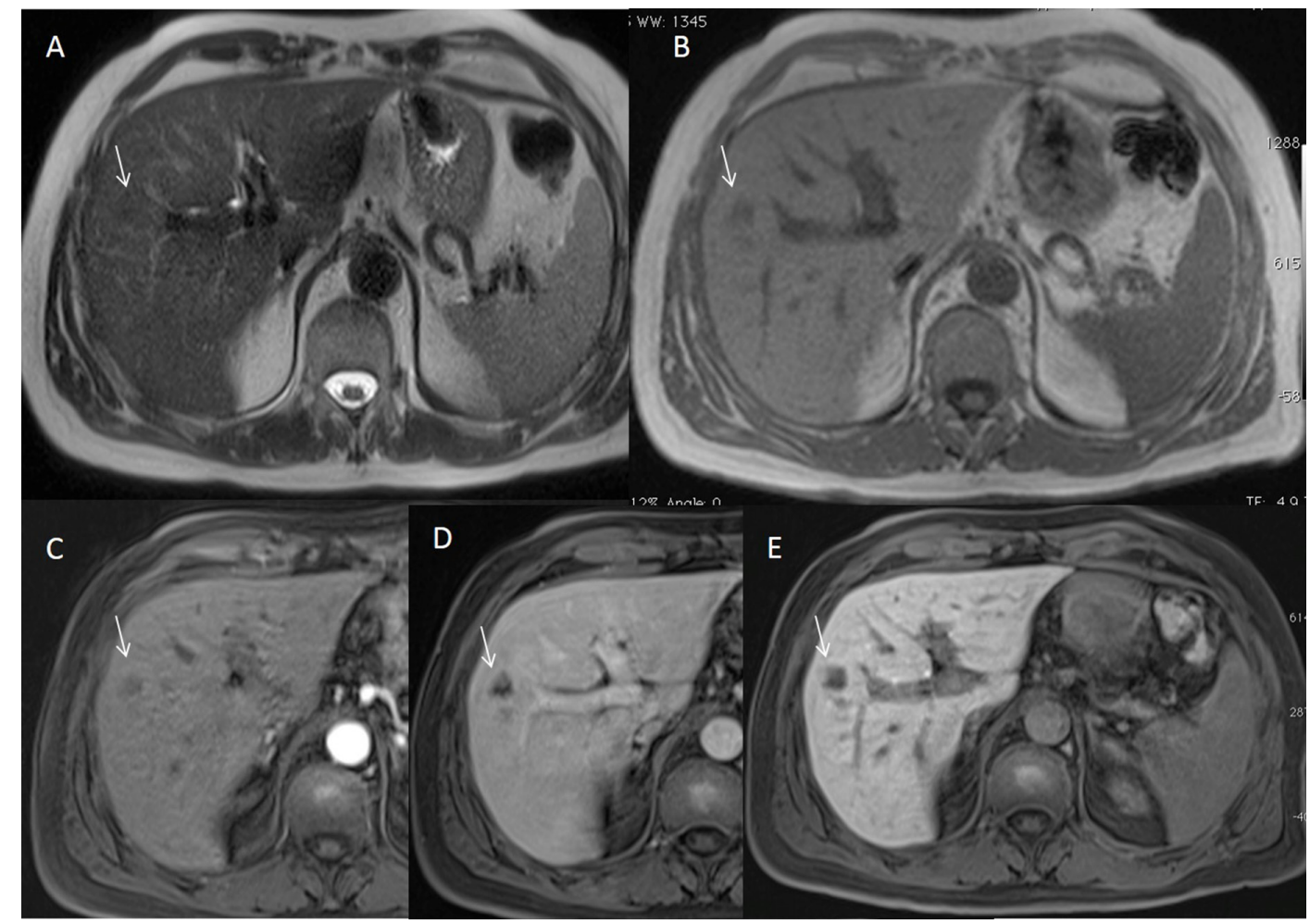

Figure 6: Man fifty-five years old with dysplastic nodule on $\mathbf{V}$ hepatic segment. The nodule is isointense (arrow) on T2-W sequence (A) and hypointense (arrow) on T1-W sequence (B). The lesion is hypointense on arterial (C), portal (D) and hepatospecific (E) phase of contrast study with EOB-GD-DTPA. 
When we analyzed the degree of hyperenhancement of arterial phase, we found that the degree was higher with Gd-BT-DO3A (a median value of 4 comparet to the median value of 2.6 with GD-EOB-DTPA, and we found no significant statistical differences among median values at MR0 and MR6 (equal to 2.6) using GD-EOB-DTPA; there was significant statistically difference between the degree of hyperenhancement of arterial phase with GdBT-DO3A and the degree of hyperenhancement of arterial phase with GD-EOB-DTPA ( $p$ value $=0.02$ at Kruskal Wallis test).

Our results are summarized in Table 1 and 2.

Table 3 reports the diagnostic accuracy for each MR imaging features. Highest accuracy was obtained by washout appearance and hypointense signal on hepatospecific phase of contrast study (97\% and 95\%, respectively).

\section{DISCUSSION}

In the management of patients with $\mathrm{HCC}$ is crucial its early diagnosis [9-13]. The identification of the vascular profile (contrast uptake in the arterial phase followed by washout in the venous phases) has permitted the non-invasive diagnostic criteria definition for $\mathrm{HCC}$ according to AASLD and EASL-EORTC guidelines. If this vascular profile is identified on dynamic CT or MR Imaging using extracellular contrast media in patients at high risk of $\mathrm{HCC}$, the diagnosis is established with the $100 \%$ of specificity [14]. However, these criteria have a sensitivity range of $50-60 \%$ in nodules smaller than 20 $\mathrm{mm}$; therefore a biopsy is still needed [15]. In this study we analyzed only nodules smaller than $20 \mathrm{~mm}$, however there is a consideration should be make. Considering that the tumor diameter is defined as the largest dimension, measured in the imaging sequence, phase, and plane in which the margins are most sharply defined, we found a difference among the lesion diameter when we analyzed different sequences, with higher difference among arterial, portal and hepatospecific phase and, although it is not significant statistically, the question born when the HCC is $10 \mathrm{~mm}$ or $20 \mathrm{~mm}$, to the bounds of the range. Moreover, the possibility to find foci of HCC in dysplastic nodules, as we established in this study, opens the question of how to measure the HCC (the entire nodule or willful part). We considered a mean value obtained from to each sequence, however it is could be considered a limit. Therefore, how

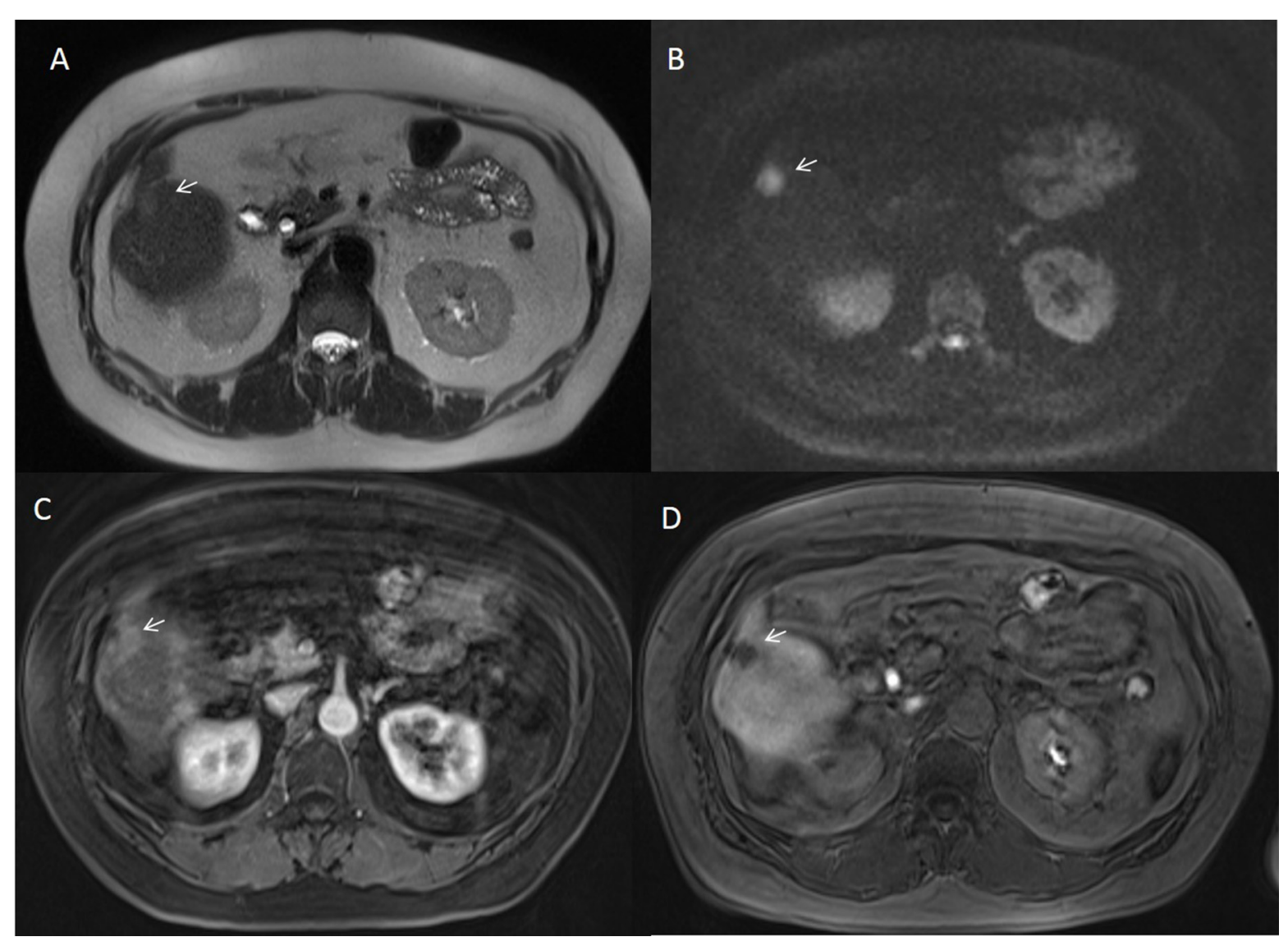

Figure 7: Woman seventy-three years old with HCC on VI hepatic segment. The HCC is hyperintense (arrow) on T2-W sequence (A) with restrict diffusion (arrow) and hyperintense signal on b800 s/mm² DW image (B). During arterial phase of contrast study (C) with EOB-GD-DTPA, the degree of image quality degradation caused by pulsatile blood flow ghost is severe (arrow). During hepatospecific phase (D) of contrast study the HCC is hypointense (arrow). 
Table 1: HCC Nodules Imaging Features

\begin{tabular}{|c|c|}
\hline Description & Numbers $(\%)$ \\
\hline $127 \mathrm{HCCs}$ & $\begin{array}{c}30(23,6 \%) \text { grade } 1,61(48,03 \%) \text { grade } 2,36(28,3 \%) \text { grade } \\
3 \text { and } 0(0 \%) \text { grade } 4\end{array}$ \\
\hline Size & $18 \mathrm{~mm}$ (range $12-20 \mathrm{~mm}$ ) \\
\hline Hyper vascular on arterial phase & $84(66,1 \%)$ \\
\hline Wash-out appearance & $124(97,6 \%)$ \\
\hline Capsule appearance & $111(87,4 \%)$ \\
\hline $\begin{array}{l}\text { Hypointense Signal on Hepatospecific phase of contrast } \\
\text { study }\end{array}$ & $127(100 \%)$ \\
\hline Hyperintense Signal on T2-W sequences & $107(84,2 \%)$ \\
\hline Restricted Diffusion & $107(84,2 \%)$ \\
\hline $\mathrm{ADC}$ & $\begin{array}{l}\text { Median value: } 1,47 \times 10^{-3} \mathrm{~mm}^{2} / \mathrm{s} \text { (range } 0,94-2,44 \times 10^{-3} \\
\mathrm{~mm}^{2} / \mathrm{s} \text { ) }\end{array}$ \\
\hline $\mathrm{fp}$ & Median value: $33,3 \%$ (range $12,14-54,08 \%$ ) \\
\hline $\mathrm{Dp}$ & $\begin{array}{l}\text { Median value: } 45,3 \times 10^{-3} \mathrm{~mm}^{2} / \mathrm{s} \text { (range } 13,7-52,7 \times 10^{-3} \\
\qquad \mathrm{~mm}^{2} / \mathrm{s} \text { ) }\end{array}$ \\
\hline Dt & Median value: $0.9 \times 10^{-3} \mathrm{~mm}^{2} / \mathrm{s}$ (range $0.81-1,51 \times 10^{-3} \mathrm{~mm}^{2} / \mathrm{s}$ ) \\
\hline \multirow[t]{2}{*}{ Quality of arterial phases } & $\begin{array}{l}\text { The median score for all arterial phase with Gd-BT-DO3A } \\
\qquad \text { (70 studies) was } 1\end{array}$ \\
\hline & $\begin{array}{c}\text { The median score for all arterial phase with GD-EOB-DTPA } \\
(140 \text { studies) was } 3 \text { (range } 1-4) \\
\text { In } 25(17,8 \%) \text { cases the images were uninterpretable }\end{array}$ \\
\hline \multirow[t]{2}{*}{ Degree of hyperenhancement of arterial phase } & The median value was 4 with Gd-BT-DO3A \\
\hline & The median value was 2.6 with GD-EOB-DTPA \\
\hline
\end{tabular}

Table 2: Dysplastic Nodules Imaging Features

\begin{tabular}{lc}
\hline Description & Numbers (\%) \\
\hline $\mathbf{2 4}$ dysplastic nodules & 18 mm (range $12-20 \mathrm{~mm})$ \\
Size & $17(70,8 \%)$ \\
Hyper vascular on arterial phase & $2(8,3 \%)$ \\
Wash-out appearance & $0(0 \%)$ \\
Capsule appearance & $7(29,16 \%)$ \\
$\begin{array}{l}\text { Hypointense Signal on Hepatospecific phase of contrast } \\
\text { study }\end{array}$ & $0(0 \%)$ \\
Hyperintense Signal on T2-W sequences & $0(0 \%)$ \\
Restricted Diffusion & The median value was 4 with Gd-BT-DO3A \\
Degree of hyperenhancement of arterial phase & The median value was 2.6 with GD-EOB-DTPA
\end{tabular}

and in which sequence to use should be standardized in order to measure the nodules according to LI-RADS, considering there are two or three categories according to the size criteria $(<20 \mathrm{~mm}$ versus $>20 \mathrm{~mm}$ for hypovascular observations and $<10 \mathrm{~mm}$ versus $10-20 \mathrm{~mm}$ versus $>20$ $\mathrm{mm}$ for hypervascular observations) [1-2]. According to our data we think that the best diagnostic performance may be obtained on hepatospecific phase when it is employed EOB-GD-DTPA, if there is not a strongly parenchymal distortion.

Arterial phase hyperenhancement is a crucial precondition to define HCC (LR-5) [1-2]. However, it is non-specific condition and may be detected in benign pathologies such as dysplastic nodules and arterioportal 
Table 3: Diagnostic Accuracy of Imaging Features

\begin{tabular}{|c|c|c|c|c|c|c|}
\hline & Sensitivity & Specificity & PPV & NPV & Accuracy & $p$ value of Fisher's exact test \\
\hline Hyper vascular on arterial phase & 66,14 & 29,17 & 83,17 & 14,00 & 60,26 & 0.8140 \\
\hline Wash-out appearance & 97,64 & 91,67 & 98,41 & 88,00 & 96,69 & 0.0001 \\
\hline Capsule appearance & 87,40 & 100,00 & 100,00 & 60,00 & 89,40 & 0.0001 \\
\hline $\begin{array}{l}\text { Hypointense Signal on } \\
\text { Hepatospecific phase of contrast } \\
\text { study }\end{array}$ & 100,00 & 70,83 & 94,78 & 100,00 & 95,36 & 0.0001 \\
\hline $\begin{array}{l}\text { Hyperintense Signal on T2-W } \\
\text { sequences }\end{array}$ & 84,25 & 100,00 & 100,00 & 54,55 & 86,75 & 0.0001 \\
\hline Restricted Diffusion & 84,25 & 100,00 & 100,00 & 54,55 & 86,75 & 0.0001 \\
\hline
\end{tabular}

Abbreviations: $\mathrm{PPV}=$ positive predictive value; NPV = negative predictive value.

Table 4: Pulse Sequence Parameters on MR studies

\begin{tabular}{lcccccc}
\hline Sequence & Orientation & $\begin{array}{c}\text { TR/TE/FA } \\
(\mathbf{m s} / \mathbf{m s} / \mathbf{d e g} \text { ) }\end{array}$ & $\begin{array}{c}\text { AT } \\
(\mathbf{m i n} .)\end{array}$ & $\begin{array}{c}\text { Acquisition } \\
\text { Matrix }\end{array}$ & $\begin{array}{c}\text { Slice thickness/ } \\
\text { Gap (mm) }\end{array}$ & Fat Suppression \\
\hline $\begin{array}{l}\text { TrueFISP } \\
\text { T2-W }\end{array}$ & Coronal & $4.30 / 2.15 / 80$ & 0.46 & $512 \times 512$ & $4 / 0$ & without \\
HASTE T2-W & Axial & $1500 / 90 / 170$ & 0.36 & $320 \times 320$ & $5 / 0$ & $\begin{array}{c}\text { Without and with } \\
\text { (SPAIR) }\end{array}$ \\
HASTE T2w & Coronal & $1500 / 92 / 170$ & 0.38 & $320 \times 320$ & $5 / 0$ & without \\
In-Out phase T1-W & Axial & $160 / 2.35 / 70$ & 0.33 & $256 \times 192$ & $5 / 0$ & without \\
DWI & Axial & $7500 / 91 / 90$ & 7 & $192 \times 192$ & $3 / 0$ & without \\
VIBE & Axial & $4.80 / 1.76 / 12$ & 0.18 & $320 \times 260$ & $3 / 0$ & with (SPAIR) \\
T1-W & & & & & &
\end{tabular}

Abbreviations: $\mathrm{W}=$ Weighted, $\mathrm{TR}=$ Repetition time, $\mathrm{TE}=$ Echo time, $\mathrm{FA}=$ Flip angle, $\mathrm{AT}=$ Acquisition time, $\mathrm{SPAIR}=$ Spectral Adiabatic Inversion Recovery, TrueFISP $=$ True fast imaging with steady state precession, HASTE = Half-Fourier acquisition single-shot turbo spin-echo, DWI $=$ Diffusion-weighted imaging, VIBE $=$ Volumetric interpolated breath hold examination.

shunts. In our series we found hyperenhancement on 17 dysplastic nodules and on $84 \mathrm{HCCs}$; hyperenhancement had a sensitivity of $66,14 \%$, a specificity of $29,17 \%$, a positive predictive value of $83,17 \%$, a negative predictive value of $14,0 \%$, a diagnostic accuracy of $60,26 \%$ ( $p$ value of 0.8140 ). Holland et al [16] demonstrated, in patients with HCC, that the majority (93\%) of hypervascular lesions on arterial phase that were occult on T2-weighted image and portal and/or equilibrium phase were non neoplastic. Conversely, Kim et al [17] demonstrated that the most significant findings associated with HCC, in nodules smaller than $20 \mathrm{~mm}$, were arterial phase hyperintensity. In our series we found that 43 HCCs were no hypervascular in arterial phase, therefore, we think that arterial phase hyperenhancement is a prerequisite but not sufficient for LR-5 classification. Another open question is which contrast medium should be used. In fact when we analyzed the degree of arterial phase hyperenhancement, we found that the degree was higher with Gd-BT-DO3A than GD-EOB-DTPA, with significant statistically difference $(\mathrm{p}$ value $=0.02$ at Kruskal Wallis test). Also, the image quality degradation was lower with Gd-BTDO3A (median score was 1) than with GD-EOB-DTPA (median score was 3 ). There was significant statistically difference between the quality on arterial phase with Gd-BT-DO3A and the quality on arterial phase with GDEOB-DTPA ( $\mathrm{p}$ value $=0.03$ at Kruskal Wallis test) and in 25 cases the images, during arterial phase with GDEOB-DTPA, were uninterpretable. These results are worthy of some considerations. First GD-EOB-DTPA is a liver-specific agent, taken up by hepatocytes [18]. It can be injected as an intravenous bolus, providing data about lesion vascularity in the different phases of contrast circulation. Additionally functional data can be obtained in the delayed, hepatobiliary phase [18]. Conversely, GdBT-DO3A is a non liver-specific agent and it provides data only about lesion vascularity, although with a better quality of arterial phase and a better hyperenhancement. Second 
our results showed that the hyperenhancement during arterial phase has a lower sensitivity, specificity, positive predictive value, negative predictive value and diagnostic accuracy than hypointensity on hepatospecific phase. Third HCC is the evolution of cirrhosis, so that when we evaluate a nodule we assessing all hepatic parenchyma in which we can found nodules in different phase of evolution or treated nodules. Considering that ablated area are evaluated according to mRECIST [19], in HCC patient we should obtain the best quality of arterial phase. In this setting we suggest to evaluate the HCC patients alternating these contrast media or considering clinic indication [10, 13, 20-21]. Washout is defined as temporal contrast-enhancement reduction relative to liver from an earlier to a later phase resulting in hypoenhancement in portal or delayed phase [22-23]. This may reveal several phenomena: rapid venous drainage, reduced portal venous supply and background liver later enhancement particularly with hepatobiliary agents [24]. Some researchers reported a variation in the timing of washout in the portal venous and delayed phases [25]. In a pilot study on enhancement of 112 histologically proven HCCs, arterial phase hypervascularity was on $74(77,96 \%)$ and portal washout within 90 seconds on $72(74,97 \%)$ in the majority of moderately differentiated HCC. However, the authors found that well-differentiated and poorly differentiated HCCs had an atypical enhancement patterns where 25 of 97 (26\%) showed washout between 91 and 180 seconds and 21 of $97(22 \%)$ showed late washout between 180 and 300 seconds [25]. In our series washout appearance was found on $97,6 \%$ of HCCs with sensitivity of $97,64 \%$, specificity of $91,67 \%$, PPV of $98,41 \%$ and NPV of $88,00 \%$ and the highest diagnostic accuracy $(96,69 \%)$ compared to other imaging feature. Our results confirmed the data by Becker et al [26], that showed as the diameter and washout criteria using a step wise LIRADS decision tree for LR3-5 observations allowed faster categorization with better inter-observer reliability while maintaining the excellent diagnostic accuracy of the most recent LI-RADS v2014. Choi et al [27] demonstrated as HCCs smaller than $15 \mathrm{~mm}$ showed typical finding of HCC less frequently than HCCs of $15 \mathrm{~mm}$ or larger in diameter. In subgroup analyses, HCCs with diameters between 10 and $15 \mathrm{~mm}$ showed similar MRI findings to HCCs with diameters of $10 \mathrm{~mm}$ or less but significantly different findings compared with HCCs with diameters from 15 to $20 \mathrm{~mm}$ and 20-30 $\mathrm{mm}$. Conversely to Choi we found that in our series (tumor with diameter between 10-20 mm), the typical hallmark without the cut-off of $15 \mathrm{~mm}$. So we think, in accordance to Becker [26], that the presence of wash-out is a crucial step wise LI-RADS decision tree for LR3-5 observations.

Capsule presence is identified as a peripheral rim of smooth hyperenhancement in the portal or delayed phase. The enhancement rim is not always a true tumor capsule, but may represent a pseudocapsule conforming to fibrous tissue and dilated sinusoids around a nodule [2223]. In our series capsule appearance was present in 111 $(89,5 \%)$ HCCs, with sensitivity of $87,40 \%$, specificity of $100 \%$, PPV of $100 \%$ and NPV of $60,0 \%$ and a diagnostic accuracy of $89,40 \%$. We no found difference between the two different contrast media, conversely to Dioguardi Burgio [28]. Our data were similar to that reported by Anis that showed as the capsule appearance has a high positive predictive value for patients at risk of HCC [29].

Threshold growth is defined as a diameter increase (a minimum of $5 \mathrm{~mm}$ and a sufficient rate). The necessary growth rate is either at least a $50 \%$ increase in diameter compared with baseline within 6 months or at least a $100 \%$ increase in diameter over more than 6 months [1-2]. In our series no HCCs showed a threshold growth while we found in a single case that a nodule at 6 months become hypervascular on arterial phase (at first MR examination it was detected only by hepatospecific phase). So we think that during the follow-up it should be considered not only the threshold growth but also the appearance of imaging features that before were not existing as hypervascular feature.

All HCCs in this study were hypointense on hepatospecific phase of contrast study, with sensitivity of $100,00 \%$, specificity of $70,83 \%$, PPV of $94,78, \mathrm{NPV}$ of 100,00 and a diagnostic accuracy of 95,36. Our results are in agreement with those reported by others [30-36]. According to Golfieri et al, during the hepatospecific phase, typical HCC and early HCC appear hypointense, while low-grade dysplastic or regenerative nodules appear as iso- or hyperintense lesions. EOB-MRI diagnostic accuracy to early HCC diagnosis was approximately 95,00$100,00 \%$ [33]. One third of hypovascular hypointense nodules in hepatospecific phase become hypervascular progressed $\mathrm{HCC}$, with a 1 and 3-year. Therefore, the authors suggested that these hypovascular nodules should be rigorously followed up or treated as typical HCC [33]. In the study by Ahn et al. [35], 9 out of 84 HCCs (10.7\%) were exclusively identified by hepatospecific phase and three were early HCCs, while in Golfieri et al [34] 19 out of 20 early HCC remained unclassified at dynamic MRI alone because of atypical behavior and were diagnosed only in the hepatospecific phase [34]. In this study 3 out of 15 nodules detected by hepatospecific phase were HCC and one became hypervascular progressed HCC, according to Golfieri [33]. So we are in agreement with Golfieri et al [36] that suggested that in atypical cirrhotic nodule, the hepatospecific phase hypointensity is the most pertinent diagnostic symbol to discriminate low-risk and high-risk nodules, since the reduction of Gd-EOB-DTPA uptake appears to occur at an early stage of hepatocarcinogenesis preceding with the portal blood flow reduction and nodule arterialization [37]. So that in hypervascular atypical HCC hepatospecific phase hypointensity can be used as the second malignancy sign. Therefore, we think that 
the hypointensity on hepatospecific phase should be considered as a major features in LI-RADS.

According to LI-RADS, T2-W hyperintensity is an ancillary imaging features. Previous study demonstrated that $\mathrm{T} 2-\mathrm{W}$ hyperintensity was a highly precise indicator of nodule malignancy, although poorly sensitive [3840], while in Golfieri et al T2-W hyperintensity was a poor predictor of malignancy in the early stages of $\mathrm{HCC}$ [36]. Conversely to Golfieri [36], Ouedraogo et al [41] demonstrated that the addition of $\mathrm{T} 2-\mathrm{W}$ hyperintensity to the AASLD criteria increased the detection rate of HCC, especially nodules smaller than $20 \mathrm{~mm}$, increased the sensitivity of MRI from $67.6 \%$ to $79 \%$. In our series $107(84,2 \%)$ out of 127 HCCs were hyperintense on T2-W sequences; all typical nodules were hyperintense on $\mathrm{T} 2-\mathrm{W}$ while $57,5 \%$ out of atypical nodules were hyperintense on $\mathrm{T} 2-\mathrm{W}$, suggesting that there was a correlation between arterialization and signal intensity on T2-W. T2-W hyperintensity showed sensitivity of 84,25 , specificity of 100,00 , PPV of 100,00, NPV of 54,55 and a diagnostic accuracy of 86,75 . In our series all lesions $\mathrm{T} 2-\mathrm{W}$ hyperintensity showed restricted diffusion with hypointense signal on ADC map (107 out of 127). So DWI showed the same sensitivity, specificity, PPV, NPV and diagnostic accuracy than T2-W. The role of DWI in HCC patient has been evaluated by different studies [42-48]. Lee et al demonstrated that the added use of DWI to the MRI with gadoxetic acid-enhanced could be a guideline to discriminate HCCs and dysplastic nodules. In their study, 86 HCCs (84.3\%) showed hyperintensity on DWI, whereas only three dysplastic nodules (13.0\%) showed this feature. So they concluded that DWI hyperintensity was extremely indicative of $\mathrm{HCC}$ in patients with chronic hepatitis or cirrhosis [44]. Also Piana et al [45] showed that arterial phase enhancement and DWI hyperintensity were more sensitive criteria for $\mathrm{HCC}$ compared to conventional criteria $(77-76 \%$ versus $60 \%$ for all HCCs and $66-60 \%$ versus $37 \%$ for HCCs smaller than $20 \mathrm{~mm}$ ). Sensitivity obtained using the arterial-dominant phase enhancement and washout (in the portal venous and/or equilibrium phases) or hyperintensity on DWI was higher $(84-85 \%$ for all HCCs and $71-74 \%$ for HCCs smaller than $20 \mathrm{~mm}$ ). In our previous study we demonstrated that that DWI could be used to predict the histological grade of $\mathrm{HCC}$; in fact we found that there was a good correlation between ADC and grading, between fp and grading, and between Dt and grading [46]. Nakanishi et al [47] showed not only the usefulness of DWI for histological grading, but also the possibility to use ADC as a preoperative prediction of early HCC recurrence within 6 months of operation. Conversely, Nasu et al [48], in a series of 125 resected HCCs (sizes range: $0.8-15 \mathrm{~cm}$ ), found no correlation between histological grade and ADC (using $b$ factors of 0 and $500 \mathrm{~s} / \mathrm{mm}^{2}$ ), although the DWI and Signal Intensity of the HCCs increased in higher grade. We found that the median value for ADC was $1,47 \times 10^{-3} \mathrm{~mm}^{2} / \mathrm{s}$ (range $0,94-2,44 \times 10^{-3} \mathrm{~mm}^{2} / \mathrm{s}$ ), for $\mathrm{fp}$ was $33,3 \%$ (range 12,14 $54,08 \%$ ), for Dp was $45,3 \times 10^{-3} \mathrm{~mm}^{2} / \mathrm{s}$ (range $13,7-52,7$ $\mathrm{x} 10^{-3} \mathrm{~mm}^{2} / \mathrm{s}$ ) and for Dt was $0.9 \times 10^{-3} \mathrm{~mm}^{2} / \mathrm{s}$ (range $0.81-1,51 \times 10^{-3} \mathrm{~mm}^{2} / \mathrm{s}$ ) with an overlapping of ADC and IVIM parameters values among typical and atypical nodules. There was not difference significant statistically between typical and atypical nodules using ADC or IVIM parameters values ( $\mathrm{p}$ value $>0.05$ at Kruskal Wallis test). These results suggested that there was not a correlation between ADC or IVIM parameters values and hypervascularization during arterial phase.

In this study we employed a $1.5 \mathrm{~T}$ MR scanner, however we think that our results could not change with use of a $3 \mathrm{~T}$ scanner. In fact, although $3 \mathrm{~T}$ systems are advantageous for musculoskeletal, neuroimaging, and angiographic applications few articles have been published regarding their use for abdominal and, particularly, liver examinations [49-50]. The quality of $3 \mathrm{~T}$ images on liver studies is reported to be equivalent to $1.5 \mathrm{~T}$ images [49], this in fact depends on the individual sequences and the particular machine [50]. Moreover, a drawback of $3 \mathrm{~T}$ is an increased number of types of artefacts. Certain imaging artefacts are more prominent at $3 \mathrm{~T}$ than at $1.5 \mathrm{~T}$, mainly because their physical parameters are dependent on the main magnetic field strength (B0): chemical shift artefacts of the first kind are directly proportional to the $\mathrm{B} 0$ and generally are twice as prominent with $3 \mathrm{~T}$ imaging, noticeable on in-phase T1-weighted images; susceptibility artefacts also increase with the $\mathrm{B} 0$ and are approximately twice as prominent at $3 \mathrm{~T}$ as compared with $1.5 \mathrm{~T}$.

\section{MATERIALS AND METHODS}

\section{Patient population}

A retrospective study, approved by National Cancer Institute Pascale Foundation of Naples, was performed through a computerized search of medical records on 160 patients underwent liver MR imaging and followed by biopsy for HCC from August 2010 to February 2017. After reviewing the medical records, 52 patients were excluded because the tumors were bigger than $2 \mathrm{~cm} ; 34$ patients because the tumors were smaller than $1 \mathrm{~cm}$ and 4 were excluded because the final pathology report was confirmed not to be HCC. The final study population included 70 patients ( 33 women and 37 men; mean age 68 years; range: 52-83 years) with 173 nodules (all with tumor diameter between 1 to $2 \mathrm{~cm}$ ). All patients had chronic liver disease which was related to hepatitis $\mathrm{C}$ virus infection in 29 patients, hepatitis B virus in 39 cases, and alcohol abuse in 2; all were stage A according to the BarcelonaClinic Liver Cancer (BCLC) classification; alphafetoprotein levels were $>4 \mathrm{ng} / \mathrm{ml}(12-320 \mathrm{ng} / \mathrm{ml}$, mean 80 $\mathrm{ng} / \mathrm{ml}$ ) in all patients. All patients underwent MR study at 0 time (MR0), after 3 (MR3) and 6 months (MR6) using 
two different contrast media (CM). Liver-specific agent, taken up by hepatocytes (Gd-EOB-BTPA), was injected at MR0 and MR6, non-specific $\mathrm{cm}$ that distributes into the vascular and extravascular extracellular spaces (Gd-BTDO3A) was injected at MR3. The mean interval standard deviation between pathologic examination and last MR study (MR6) was 15 days (range 4-28 days).

\section{MR imaging protocol}

MR imaging was performed by using a 1.5T MR (Magnetom Symphony, with Total Imaging Matrix Package, Siemens, Erlangen, Germany) with 8-element body and phased array coils. The MRI examination consisted of basal images taken before IV administration of contrast medium and then functional dynamic sequences obtained after IV injection of $\mathrm{cm}$, acquiring the last series of images, when we used hepatospecific $\mathrm{cm}$, with a delay of 20 minutes during the hepatobiliary excretion of the $\mathrm{cm}$. The baseline sequences obtained before IV contrast medium were coronal True fast imaging with steady state precession (TrueFISP) T2-weighted free breathing; axial Half-Fourier Acquisition Single-Shot Turbo Spin-Echo (HASTE) T2-weighted, with controlled respiration, without and with fat-suppressed (FS) gradientecho pulse; coronal HASTE T2-weighted, without FS; axial flash in-out phase T1-weighted, with controlled respiration; Volumetric Interpolated Breath-hold Examination (VIBE) T1-weighted SPAIR with controlled respiration; diffusion weighted imaging (DWI) with planar echo-pulse sequence (EPI) at several b value $b$ value 0,50 , 100, 200, 400, 600, and $800 \mathrm{~s} / \mathrm{mm}^{2}$. As liver-specific CM, the EOB-Gd-BPTA (Primovist, Bayer Schering Pharma, Germany) was employed. All patients received $0.1 \mathrm{ml} / \mathrm{kg}$ of EOB-Gd-BPTA by means of a power injector (Spectris Solaris ${ }^{\circledR}$ EP MR, MEDRAD Inc., Indianola, IA, USA), at an infusion rate of $1 \mathrm{ml} / \mathrm{s}$. As non-specific agent the GdBT-DO3A (Gadovist, Bayer Schering Pharma, Germany) was employed. All patients received $0.1 \mathrm{ml} / \mathrm{kg}$ of Gd-BTDO3A by means of a power injector (Spectris Solaris ${ }^{\circledR}$ EP MR, MEDRAD Inc., Indianola, IA, USA), at an infusion rate of $2 \mathrm{ml} / \mathrm{s}$. After contrast medium administration, VIBE T1-weighted FS (SPAIR) sequences were acquired in different phases: hepatic arterial (35 s delay), portal venous (90 s), equilibrium (120 s), and hepatobiliary excretion (20 minutes). Details of sequence parameters were reported in Table 4.

\section{Images analysis}

Three expert hepatic radiologists retrospectively and independently reviewed all images and served as the consensus. The observers were blinded to clinical history and previous imaging studies. We analyzed only lesion smaller than $2 \mathrm{~cm}$ and greater than $1 \mathrm{~cm}$. We used LI-RADS v2014 classification and histological analysis served as the standard of reference.
For each nodule we recorded, according to LIRADS classification, the size, the presence and quality of arterial-phase hyperenhancement, washout appearance, capsule appearance, threshold growth. Additionally, we recorded signal intensity (SI) on T2-W images, on DWI, on maps of apparent diffusion coefficient (ADC) and, when we used EOB-GD-BPTA, SI on T1-W images during hepatospecific phase on contrast study. For each nodule, we reported the median ADC value and median values of Intravoxel incoherent motion (IVIM) related parameters of pseudo-diffusivity (Dp), perfusion fraction (fp), and tissue diffusivity (Dt).

When we found arterial-phase hyperenhancement, washout and capsule appearance, we defined the HCC as typical. When we found, washout and capsule appearance, but not the arterial-phase hyperenhancement, we defined the HCC as atypical.

The lesion was categorized hyperintense, isointense or hypointense relative to the surrounding liver parenchyma. The lesions that were seen only as hypointense on the hepatobiliary phase were graded as LI-RADS 3.

For lesion detection with DWI, the observers analyzed all $b$ values.

Each observer independently evaluated the presence of arterial phase hyperenhancement using a four-point scale $(1=$ absent, 2 = low intensity, $3=$ mild intensity, $4=$ high intensity), to compare the efficacy of the two different contrast media to evaluate hyperenhancement.

Also, each observer independently evaluated the degree of image quality degradation caused by respiratory ghost, pulsatile blood flow ghost, and susceptibility artifacts using a four-point scale $(1=$ absent or minimal, $2=$ mild, 3 = moderate, $4=$ severe). A "severe" score indicated that an image was uninterpretable and a "mild" score indicated that the artifact did not affect interpretation.

A consensus read was performed when there was disagreement between the readers.

In this study the diffusion parameters estimation was performed using the intravoxel incoherent motion method [6-7].

Bi-exponential model to estimate the IVIM-related parameters of pseudo-diffusivity $\left(\mathrm{D}_{\mathrm{p}}\right)$, perfusion fraction $\left(f_{p}\right)$, and tissue diffusivity $\left(D_{t}\right)$ was described by the following equation

$$
\frac{S_{0}}{S_{b}}=\mathrm{f}_{\mathrm{p} .} \exp \left(-\mathrm{b} \cdot \mathrm{D}_{\mathrm{p}}\right)+\left(1-\mathrm{f}_{\mathrm{p}}\right) \cdot \exp \left(-b \cdot D_{t}\right)
$$

We used a VARiable PROjection approach to estimate the three parameters because the bi-exponential model may often be ill-conditioned because of a limited number of samples, small perfusion fraction and/or similar compartmental diffusivities. In a previous study, we have demonstrated that the VARiable PROjection algorithm is superior to the conventional Levenberg-Marquardt 
algorithm for non linear curve fitting in intravoxel incoherent motion method for DW-MRI data analysis [8]. A brief explanation of VARPRO approach is described in the following.

Rearranging the equation (1) the $S(b) / S_{0}-e^{-b D_{t}}$ is the product of $f$ and a nonlinear function of $\mathrm{Dt}$ and $\mathrm{Dp}$ :

$$
\mathrm{f}\left(\mathrm{D}_{\mathrm{p}} ; \mathrm{D}_{\mathrm{t}} ; \mathrm{b}\right)=S(b) / S_{0}-e^{-b D_{t}}=f\left(e^{-b D_{p}}-e^{-b D_{t}}\right)
$$

Letting $f\left(D_{p} ; D_{t} ; b\right)$ the cost functional becomes:

$$
S(b) / S_{0} \cdot-e^{-b D_{t}}=\left\|\mathrm{y}-\mathrm{f}\left(\mathrm{D}_{\mathrm{p}} ; \mathrm{D}_{\mathrm{t}} ; \mathrm{b}\right) \mathrm{f}\right\|_{2}
$$

Therefore, a separable nonlinear least square model known as VARiable PROjection (VARPRO) can be used to calculate the diffusion parameters. If we knew, the estimate of the nonlinear parameters Dp and Dt the estimate of the linear parameter $f$ could be obtained by:

$$
f=e^{-b D_{t}}+\mathrm{y}+\mathrm{f}\left(\mathrm{D}_{\mathrm{p}} ; \mathrm{D}_{\mathrm{t}} ; \mathrm{b}\right)^{+}
$$

where $f\left(D_{p} ; D_{t} ; b\right)^{+}$is the Moore-Penrose generalized inverse of $f\left(D_{p} ; D_{t} ; b\right)$. Therefore, a new cost functional can be constructed:

$$
S(b) / S_{0} .-e^{-b D_{t}}=\left\|\mathrm{y}-\mathrm{f}\left(\mathrm{D}_{\mathrm{p}} ; \mathrm{D}_{\mathrm{t}} ; \mathrm{b}\right) \mathrm{f}\left(\mathrm{D}_{\mathrm{p}} ; \mathrm{D}_{\mathrm{t}} ; \mathrm{b}\right)^{+} \mathrm{y}\right\|_{2}
$$

This analysis was ROI-based using median value of single voxel signals for each $b$ value. ROIs for the tumor were manually drawn to include such hyperintense voxels on image at b value $800 \mathrm{~s} / \mathrm{mm}^{2}$. No motion correction algorithm was used but ROIs were drawn taking care to exclude areas in which movement artifacts or blurring caused voxel misalignments.

The data analysis was performed using an in-house software written in Matlab (The MathWorks, Inc., Natick, MA, USA).

\section{Statistical analysis}

Data were expressed in terms of median value \pm range. Kruskal Wallis non-parametric test was performed to emphasize significant statistically difference between median value in different population subgroups. Sensitivity, specificity, positive predictive value (PPV), negative predictive value (NPV) and accuracy were assessed. Fisher's exact test was used to evaluate statistical significance of dichotomous $2 \times 2$ tables. A p value $<0.05$ was considered statistically significant. All analyses were performed using Statistics Toolbox of Matlab R2007a (The Math-Works Inc., Natick, MA).

\section{CONCLUSION}

LI-RADS is a system score to interpret and report imaging features in patients at risk for HCC. Although the arterial phase hyperenhancement is an essential prerequisite for definitely HCC, it not sufficient for LR-5 categorization. Moreover the degree of hyperenhancement is higher with Gd-BT-DO3A than GD-EOB-DTPA, so as the image quality degradation was lower with Gd-BTDO3A compared to GD-EOB-DTPA. However considering that the hyperenhancement during arterial phase has a lower sensitivity, specificity, PPV, NPV and diagnostic accuracy than hypointensity on hepatospecific phase and that hypointensity in the hepatospecific phase and washout appearance are the most significant diagnostic signs to discriminate low-risk from high-risk nodules, patients at risk for HCC may be evaluated alternating these contrast media. The capsule appearance, T2-W hyperintensity and restricted diffusion have a high positive predictive value for HCC while threshold growth may be associated to other features as the appearance of arterial phase hyperenhancement.

\section{ACKNOWLEDGMENTS}

The authors are grateful to Alessandra Trocino, librarian at the National Cancer Institute of Naples, Italy. Moreover, for the collaboration, authors are grateful to Maria Bruno, Laura Galeani, Rita Guarino, Leandro Eto and Assunta Zazzaro.

\section{CONFLICTS OF INTEREST}

The authors do not have any conflicts of interest.

\section{REFERENCES}

1. An C, Rakhmonova G, Choi JY, Kim MJ. Liver imaging reporting and data system (LI-RADS) version 2014: understanding and application of the diagnostic algorithm. Clin Mol Hepatol. 2016; 22:296-307.

2. Tang A, Valasek MA, Sirlin CB. Update on the Liver Imaging Reporting and Data System: What the Pathologist Needs to Know. Adv Anat Pathol. 2015; 22:314-22.

3. European Association for Study of LiverEuropean Organisation for Research and Treatment of Cancer. EASL-EORTC clinical practice guidelines: management of hepatocellular carcinoma. Eur J Cancer. 2012; 48:599-641.

4. Santillan CS, Tang A, Cruite I, Shah A, Sirlin CB. Understanding LI-RADS: a primer for practical use. Magn Reson Imaging Clin N Am. 2014; 22:337-52.

5. American College of Radiology. Liver Imaging Reporting and Data System version 2014. Accessed April 15 2016, from http://www.acr.org/Quality-Safety/Resources/LIRADS.

6. Le Bihan D, Breton E, Lallemand D, Grenier P, Cabanis E, Laval-Jeantet M. MR imaging of intravoxel incoherent motions: application to diffusion and perfusion in neurologic disorders. Radiology. 1986; 161:401-07.

7. Le Bihan D, Breton E, Lallemand D, Aubin ML, Vignaud J, Laval-Jeantet M. Separation of diffusion and perfusion in intravoxel incoherent motion MR imaging. Radiology. $1988 ; 168: 497-505$. 
8. Fusco R, Sansone M, Petrillo A. The Use of the LevenbergMarquardt and Variable Projection Curve-Fitting Algorithm in Intravoxel Incoherent Motion Method for DW-MRI Data Analysis. Appl Magn Reson. 2015; 46:551-58.

9. Granata V, Fusco R, Catalano O, Piccirillo M, De Bellis M, Izzo F, Petrillo A. Percutaneous ablation therapy of hepatocellular carcinoma with irreversible electroporation: MRI findings. AJR Am J Roentgenol. 2015; 204:1000-07.

10. Granata V, de Lutio di Castelguidone E, Fusco R, Catalano O, Piccirillo M, Palaia R, Izzo F, Gallipoli AD, Petrillo A. Irreversible electroporation of hepatocellular carcinoma: preliminary report on the diagnostic accuracy of magnetic resonance, computer tomography, and contrast-enhanced ultrasound in evaluation of the ablated area. Radiol Med (Torino). 2016; 121:122-31.

11. Izzo F, Palaia R, Albino V, Amore A, di Giacomo R, Piccirillo M, Leongito M, Nasto A, Granata V, Petrillo A, Lastoria S. Hepatocellular carcinoma and liver metastases: clinical data on a new dual-lumen catheter kit for surgical sealant infusion to prevent perihepatic bleeding and dissemination of cancer cells following biopsy and locoregional treatments. Infect Agent Cancer. 2015; 10:11.

12. Izzo F, Albino V, Palaia R, Piccirillo M, Tatangelo F, Granata V, Petrillo A, Lastoria S. Hepatocellular carcinoma: preclinical data on a dual-lumen catheter kit for fibrin sealant infusion following loco-regional treatments. Infect Agent Cancer. 2014; 9:39.

13. Granata V, Petrillo M, Fusco R, Setola SV, de Lutio di Castelguidone E, Catalano O, Piccirillo M, Albino V, Izzo F, Petrillo A. Surveillance of HCC Patients after Liver RFA: Role of MRI with Hepatospecific Contrast versus ThreePhase CT Scan-Experience of High Volume Oncologic Institute. Gastroenterol Res Pract. 2013; 2013:469097.

14. Bruix J, Sherman M, and American Association for the Study of Liver Diseases. Management of hepatocellular carcinoma: an update. Hepatology. 2011; 53:1020-22.

15. Forner A, Vilana R, Ayuso C, Bianchi L, Solé M, Ayuso JR, Boix L, Sala M, Varela M, Llovet JM, Brú C, Bruix J. Diagnosis of hepatic nodules $20 \mathrm{~mm}$ or smaller in cirrhosis: prospective validation of the noninvasive diagnostic criteria for hepatocellular carcinoma. Hepatology. 2008; 47:97-104.

16. Holland AE, Hecht EM, Hahn WY, Kim DC, Babb JS, Lee VS, West AB, Krinsky GA. Importance of small $(<$ or $=20-\mathrm{mm})$ enhancing lesions seen only during the hepatic arterial phase at MR imaging of the cirrhotic liver: evaluation and comparison with whole explanted liver. Radiology. 2005; 237:938-44.

17. Kim TK, Lee KH, Jang HJ, Haider MA, Jacks LM, Menezes RJ, Park SH, Yazdi L, Sherman M, Khalili K. Analysis of gadobenate dimeglumine-enhanced MR findings for characterizing small $(1-2-\mathrm{cm})$ hepatic nodules in patients at high risk for hepatocellular carcinoma. Radiology. 2011; 259:730-38.

18. Granata V, Cascella M, Fusco R, dell'Aprovitola N, Catalano O, Filice S, Schiavone V, Izzo F, Cuomo A,
Petrillo A. Immediate adverse reactions to gadoliniumbased MR contrast media: a retrospective analysis on 10,608 examinations. Biomed Res Int. 2016; 2016:3918292.

19. Lencioni R, Llovet JM. Modified RECIST (mRECIST) assessment for hepatocellular carcinoma. Semin Liver Dis. 2010; 30:52-60.

20. Schelhorn J, Best J, Dechêne A, Göbel T, Bertram S, Lauenstein T, Kinner S. Evaluation of combined Gd-EOBDTPA and gadobutrol magnetic resonance imaging for the prediction of hepatocellular carcinoma grading. Acta Radiol. 2016; 57:932-38.

21. Schelhorn J, Best J, Reinboldt MP, Gerken G, Ruhlmann M, Lauenstein TC, Antoch G, Kinner S. Therapy response assessment after radioembolization of patients with hepatocellular carcinoma — comparison of MR imaging with gadolinium ethoxybenzyl diethylenetriamine penta-acetic acid and gadobutrol. J Vasc Interv Radiol. 2015; 26:972-79.

22. Park YN, Kim MJ. Hepatocarcinogenesis: imagingpathologic correlation. Abdom Imaging. 2011; 36:232-43.

23. Efremidis SC, Hytiroglou P. The multistep process of hepatocarcinogenesis in cirrhosis with imaging correlation. Eur Radiol. 2002; 12:753-64.

24. Hope TA, Fowler KJ, Sirlin CB, Costa EA, Yee J, Yeh BM, Heiken JP. Hepatobiliary agents and their role in LI-RADS. Abdom Imaging. 2015; 40:613-25.

25. Jang HJ, Kim TK, Burns PN, Wilson SR. Enhancement patterns of hepatocellular carcinoma at contrast-enhanced US: comparison with histologic differentiation. Radiology. 2007; 244:898-906.

26. Becker AS, Barth BK, Marquez PH, Donati OF, Ulbrich EJ, Karlo C, Reiner CS, Fischer MA. Increased interreader agreement in diagnosis of hepatocellular carcinoma using an adapted LI-RADS algorithm. Eur J Radiol. 2017; 86:33-40.

27. Choi MH, Choi JI, Lee YJ, Park MY, Rha SE, Lall C. MRI of Small Hepatocellular Carcinoma: Typical Features Are Less Frequent Below a Size Cutoff of $1.5 \mathrm{~cm}$. AJR Am J Roentgenol. 2016; 27:1-8.

28. Dioguardi Burgio M, Picone D, Cabibbo G, Midiri M, Lagalla R, Brancatelli G. MR-imaging features of hepatocellular carcinoma capsule appearance in cirrhotic liver: comparison of gadoxetic acid and gadobenate dimeglumine. Abdom Radiol (NY). 2016; 41:1546-54.

29. Anis M. Imaging of hepatocellular carcinoma: new approaches to diagnosis. Clin Liver Dis. 2015; 19:325-40.

30. Tong HF, Liang HB, Mo ZK, Guan TP, Yang J, Fang $\mathrm{CH}$. Quantitative analysis of gadoxetic acid-enhanced magnetic resonance imaging predicts histological grade of hepatocellular carcinoma. Clin Imaging. 2017; 43:9-14.

31. Jeon I, Cho ES, Kim JH, Kim DJ, Yu JS, Chung JJ. Feasibility of 10-Minute Delayed Hepatocyte Phase Imaging Using a $30^{\circ}$ Flip Angle in Gd-EOB-DTPAEnhanced Liver MRI for the Detection of Hepatocellular Carcinoma in Patients with Chronic Hepatitis or Cirrhosis. PLoS One. 2016; 11:e0167701. 
32. Toyoda H, Kumada T, Tada T, Sone Y, Maeda A, Kaneoka Y. Non-hypervascular hypointense nodules on Gd-EOBDTPA-enhanced MRI as a predictor of outcomes for earlystage HCC. Hepatol Int. 2015; 9:84-92.

33. Golfieri R, Garzillo G, Ascanio S, Renzulli M. Focal lesions in the cirrhotic liver: their pivotal role in gadoxetic acidenhanced MRI and recognition by the Western guidelines. Dig Dis. 2014; 32:696-704.

34. Golfieri R, Renzulli M, Lucidi V, Corcioni B, Trevisani F, Bolondi L. Contribution of the hepatobiliary phase of Gd-EOB-DTPA-enhanced MRI to Dynamic MRI in the detection of hypovascular small $(\leq 2 \mathrm{~cm}) \mathrm{HCC}$ in cirrhosis. Eur Radiol. 2011; 21:1233-42.

35. Ahn SS, Kim MJ, Lim JS, Hong HS, Chung YE, Choi JY. Added value of gadoxetic acid-enhanced hepatobiliary phase MR imaging in the diagnosis of hepatocellular carcinoma. Radiology. 2010; 255:459-66.

36. Golfieri R, Grazioli L, Orlando E, Dormi A, Lucidi V, Corcioni B, Dettori E, Romanini L, Renzulli M. Which is the best MRI marker of malignancy for atypical cirrhotic nodules: hypointensity in hepatobiliary phase alone or combined with other features? Classification after Gd-EOBDTPA administration. J Magn Reson Imaging. 2012; 36:648-57.

37. Kogita S, Imai Y, Okada M, Kim T, Onishi H, Takamura M, Fukuda K, Igura T, Sawai Y, Morimoto O, Hori M, Nagano $\mathrm{H}$, Wakasa K, et al. Gd-EOB-DTPA-enhanced magnetic resonance images of hepatocellular carcinoma: correlation with histological grading and portal blood flow. Eur Radiol. 2010; 20:2405-13.

38. Kelekis NL, Semelka RC, Worawattanakul S, de Lange EE, Ascher SM, Ahn IO, Reinhold C, Remer EM, Brown JJ, Bis KG, Woosley JT, Mitchell DG. Hepatocellular carcinoma in North America: a multiinstitutional study of appearance on T1-weighted, T2-weighted, and serial gadoliniumenhanced gradient-echo images. AJR Am J Roentgenol. 1998; 170:1005-13.

39. Willatt JM, Hussain HK, Adusumilli S, Marrero JA. MR Imaging of hepatocellular carcinoma in the cirrhotic liver: challenges and controversies. Radiology. 2008; 247:311-30.

40. Kim JI, Lee JM, Choi JY, Kim YK, Kim SH, Lee JY, Han JK, Choi BI. The value of gadobenate dimeglumineenhanced delayed phase MR imaging for characterization of hepatocellular nodules in the cirrhotic liver. Invest Radiol. 2008; 43:202-10.

41. Ouedraogo W, Tran-Van Nhieu J, Baranes L, Lin SJ, Decaens T, Laurent A, Djabbari M, Pigneur F, Duvoux C, Kobeiter H, Deux JF, Rahmouni A, Luciani A. [Evaluation of noninvasive diagnostic criteria for hepatocellular carcinoma on pretransplant MRI (2010): correlation between MR imaging features and histological features on liver specimen]. [Article in French]. J Radiol. 2011; 92:688-700.

42. Shankar S, Kalra N, Bhatia A, Srinivasan R, Singh P, Dhiman RK, Khandelwal N, Chawla Y. Role of Diffusion Weighted Imaging (DWI) for Hepatocellular Carcinoma (HCC) Detection and its Grading on 3T MRI: A Prospective Study. J Clin Exp Hepatol. 2016; 6:303-10.

43. Xu PJ, Yan FH, Wang JH, Shan Y, Ji Y, Chen CZ. Contribution of diffusion-weighted magnetic resonance imaging in the characterization of hepatocellular carcinomas and dysplastic nodules in cirrhotic liver. J Comput Assist Tomogr. 2010; 34:506-12.

44. Lee MH, Kim SH, Park MJ, Park CK, Rhim H. Gadoxetic acid-enhanced hepatobiliary phase MRI and high-bvalue diffusion-weighted imaging to distinguish welldifferentiated hepatocellular carcinomas from benign nodules in patients with chronic liver disease. AJR Am J Roentgenol. 2011; 197:W868-75.

45. Piana G, Trinquart L, Meskine N, Barrau V, Beers BV, Vilgrain V. New MR imaging criteria with a diffusionweighted sequence for the diagnosis of hepatocellular carcinoma in chronic liver diseases. J Hepatol. 2011; 55:126-32.

46. Granata V, Fusco R, Catalano O, Guarino B, Granata F, Tatangelo F, Avallone A, Piccirillo M, Palaia R, Izzo F, Petrillo A. Intravoxel incoherent motion (IVIM) in diffusionweighted imaging (DWI) for Hepatocellular carcinoma: correlation with histologic grade. Oncotarget. 2016; 7:79357-64. https://doi.org/10.18632/oncotarget.12689.

47. Nakanishi M, Chuma M, Hige S, Omatsu T, Yokoo H, Nakanishi K, Kamiyama T, Kubota K, Haga H, Matsuno Y, Onodera Y, Kato M, Asaka M. Relationship between diffusion-weighted magnetic resonance imaging and histological tumor grading of hepatocellular carcinoma. Ann Surg Oncol. 2012; 19:1302-09.

48. Nasu K, Kuroki Y, Tsukamoto T, Nakajima H, Mori K, Minami M. Diffusion-weighted imaging of surgically resected hepatocellular carcinoma: imaging characteristics and relationship among signal intensity, apparent diffusion coefficient, and histopathologic grade. AJR Am J Roentgenol. 2009; 193:438-44.

49. von Falkenhausen MM, Lutterbey G, Morakkabati-Spitz N, Walter O, Gieseke J, Blömer R, Willinek WA, Schild HH, Kuhl CK. High-field-strength MR imaging of the liver at 3.0 T: intraindividual comparative study with MR imaging at 1.5 T. Radiology. 2006; 241:156-66.

50. Ramalho M, Altun E, Herédia V, Zapparoli M, Semelka R. Liver MR imaging: 1.5T versus 3T. Magn Reson Imaging Clin N Am. 2007; 15:321-47, vi. 